\title{
Imaging Subsurface Reflection Phase with Quantized Electrons
}

\section{Citation}

Altfeder, I. B., V. Narayanamurti, and D. M. Chen. 2002. “Imaging Subsurface Reflection Phase with Quantized Electrons." Physical Review Letters 88 (20). https://doi.org/10.1103/ physrevlett.88.206801.

\section{Permanent link}

http://nrs.harvard.edu/urn-3:HUL.InstRepos:41417281

\section{Terms of Use}

This article was downloaded from Harvard University's DASH repository, and is made available under the terms and conditions applicable to Other Posted Material, as set forth at http:// nrs.harvard.edu/urn-3:HUL.InstRepos:dash.current.terms-of-use\#LAA

\section{Share Your Story}

The Harvard community has made this article openly available.

Please share how this access benefits you. Submit a story.

Accessibility 


\title{
Imaging Subsurface Reflection Phase with Quantized Electrons
}

\author{
I. B. Altfeder, ${ }^{1,2}$ V. Narayanamurti, ${ }^{1}$ and D. M. Chen ${ }^{2}$ \\ ${ }^{1}$ Gordon McKay Laboratory, Harvard University, Cambridge, Massachusetts 02138 \\ ${ }^{2}$ Rowland Institute for Science, Cambridge, Massachusetts 02142
}

(Received 27 November 2001; published 3 May 2002)

\begin{abstract}
Lead quantum wells $(\mathrm{QW})$ epitaxially grown on annealed $\mathrm{Pb} / \mathrm{Si}(111)$ interface form a model system for the study of interactions between quantized electrons and adiabatically modulated boundaries. Tunnel spectra of this system reveal a previously unknown adiabatic shift of QW resonances due to lateral variations of the electronic reflection phase at the buried interface. With this effect, lateral distribution of the subsurface reflection phase can be probed, using scanning tunneling microscopy.
\end{abstract}

DOI: 10.1103/PhysRevLett.88.206801

PACS numbers: 73.21.Cd, 68.37.-d, 73.22.-f

Boundary conditions are the important elements in any quantum-mechanical problem. As the size of the system reduces, the surface-to-volume ratio increases rapidly, and it is widely accepted that the boundary conditions should play a crucial role [1-4].

Recent advances in bottom-up nanoengineering have introduced a fascinating class of coherent electronic systems, which are based on nanoscale self-organization during heteroepitaxial growth. This new class of coherent electronic systems includes self-assembled quantum dots [5], magnetic nanostructures [6], nanodot arrays [7], and quantum wedge [8]. Quantum phase coherence in heteroepitaxial systems has already shown to be a powerful tool for the study of nanoscale subsurface objects $[9,10]$. One such example has recently been demonstrated in thin $\mathrm{Pb}(111)$ quantum wells $(\mathrm{QW})$ epitaxially grown on $7 \times 7$ reconstructed $\mathrm{Si}(111)$ [10]. Since the Pb Fermi surface [11] (FS) in a 2nd Brillouin zone is composed of flat (111) faces (nested), the electron group velocities are directed along the crystal orientations [111]. Therefore, a significant portion of electrons, which are confined in a thin $\mathrm{Pb}(111)$ film, oscillate between its opposite boundaries and almost do not exhibit lateral motion (FS nesting-driven localization). This phenomenon allows direct imaging of nanoscale subsurface objects, using a conventional scanning tunneling microscopy (STM). Specific to the $7 \times 7$ superlattice is the periodic arrangement of triangular domains, separated by atomically narrow dimer rows. Within the domains, the buried interface acts as a perfectly flat electron mirror, providing the necessary boundary conditions for the observation of a quantum-size effect (QSE). In contrast, the buried dimer rows and corner holes serve as strong subsurface scatterers (see Fig. 1a), which introduce strong nonadiabatic effects and locally (within half the Fermi wavelength $\lambda_{f} / 2 \approx 6 \AA$ ) suppress the QSE. In the opposite limit, one can imagine an atomically smooth interface, which is composed of a slowly varying boundary potential, free of strong scattering and hence acting as an adiabatically modulated electron mirror, as suggested in Fig. 1b.

In this Letter, we present an experimental study of this new regime, using the same heteroepitaxial system
$\mathrm{Pb} / \mathrm{Si}(111)$ prepared under a different procedure. Our results reveal the previously unknown spectral shift of the $\mathrm{QW}$ resonances, which we attribute to the electron reflection phase at the buried interface.

As was shown in previous studies [12-14], epitaxial growth of the $\mathrm{Pb}$ films on $\mathrm{Si}(111)$ yields two distinct interfaces. In both cases, $\mathrm{Pb}$ forms (111) oriented 3D crystallites following the formation of the wetting layers. When the $\mathrm{Pb}$ atoms are deposited at room temperature, the $7 \times 7$ reconstruction of the silicon surface remains unaltered even when buried below the $3 \mathrm{D} \mathrm{Pb}$ islands. In contrast, postannealing to more than $300{ }^{\circ} \mathrm{C}$ or deposition of $\mathrm{Pb}$ atoms at the elevated substrate temperatures removes the $7 \times 7$ reconstruction. In this phase, the first monolayer of $\mathrm{Pb}$ atoms develops a closely packed interface with the $\mathrm{Si}(111)$ substrate, while the rest of the metallic layers possess the crystal lattice of bulk $\mathrm{Pb}$. The lattice spacing mismatch

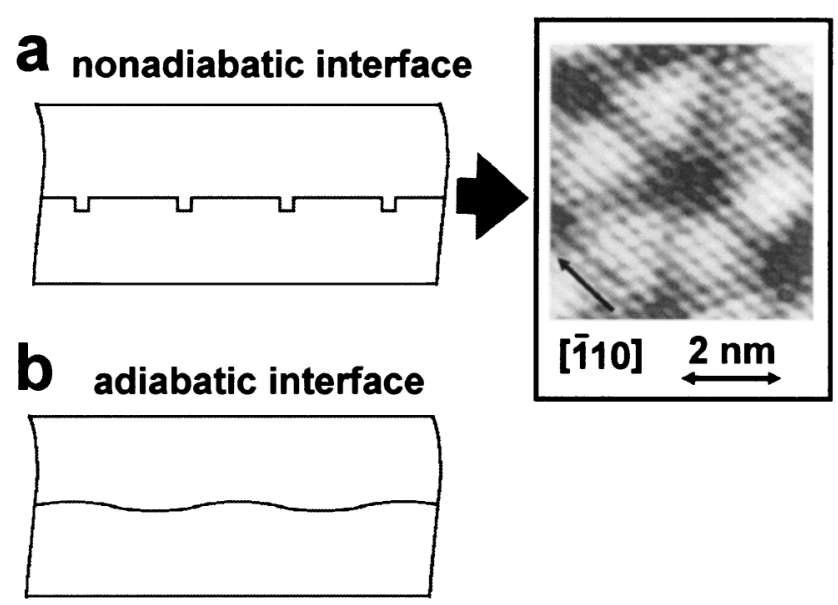

FIG. 1. The two distinct types of buried interfaces, corresponding to the two epitaxial phases of $\mathrm{Pb}$ on $\mathrm{Si}(111)$. (a) Atomically rough interface (nonadiabatic regime), which corresponds to a buried $7 \times 7$ phase of $\mathrm{Si}(111)$. Inset: electronic image of such an interface, similar to one reported in Ref. [10]. On the background of the $\mathrm{Pb}$ atomic lattice, STM reveals the subsurface scattering potential. (b) Atomically smooth interface (adiabatic regime), which corresponds to an annealed phase of $\mathrm{Pb}$ on $\operatorname{Si}(111)$ 
(3.85 $\AA$ for $\mathrm{Si}$ and $3.50 \AA$ for $\mathrm{Pb}$ ) causes a rotation of the $\mathrm{Pb}(111)$ islands with respect to the $\mathrm{Si}(111)$ plane. As a result, the annealed interface is structurally smoother than the nonannealed. Thus, the utilization of the two epitaxial phases of $\mathrm{Pb}$ on $\mathrm{Si}(111)$ makes it possible to design two different types of $\mathrm{Pb} \mathrm{QW}$ and to study the interactions of the quantized electrons both with atomically rough [10] and with the atomically smooth buried interface, presented herein.

The experimental setup consisted of a dual-chamber ultrahigh vacuum (UHV) system, equipped with standard tools for surface preparation and analysis and with lowtemperature STM. The silicon substrate was cleaned inside the sample preparation chamber by a sequence of ion beam sputtering and direct current heating to $1100{ }^{\circ} \mathrm{C}$. After cleaning, a monolayer of $\mathrm{Pb}$ atoms was deposited from an effusion cell onto the $7 \times 7$ reconstructed $\mathrm{Si}(111)$ surface. The sample was then annealed to $350^{\circ} \mathrm{C}$ in order to eliminate the $7 \times 7$ reconstruction. Next, the $3 \mathrm{D} \mathrm{Pb}$ islands were grown by depositing additional $\mathrm{Pb}$ atoms onto the cold substrate. The sample was then transferred in situ into the low temperature UHV STM.

In Fig. 2, we show a typical STM image obtained on a $\mathrm{Pb}$ island at $77 \mathrm{~K}$. The $\mathrm{Pb}$ islands grow in a wedge-shaped geometry with a flat (111) top surface, while the thickness changes due to atomic steps on the substrate. The sequence of nearly parallel lines, crossing the image (from top to bottom), represent the electron interference fringes [8], which result from the QSE and depict the regions of constant $\mathrm{Pb}$ thickness. In addition, within each constant-height partition of the $\mathrm{Pb}$ island, the STM reveals the hexagonal superlattice with a period of $40 \AA$. Although the Pb thickness in Fig. 2 increases by 7 atomic layers (from left to right),

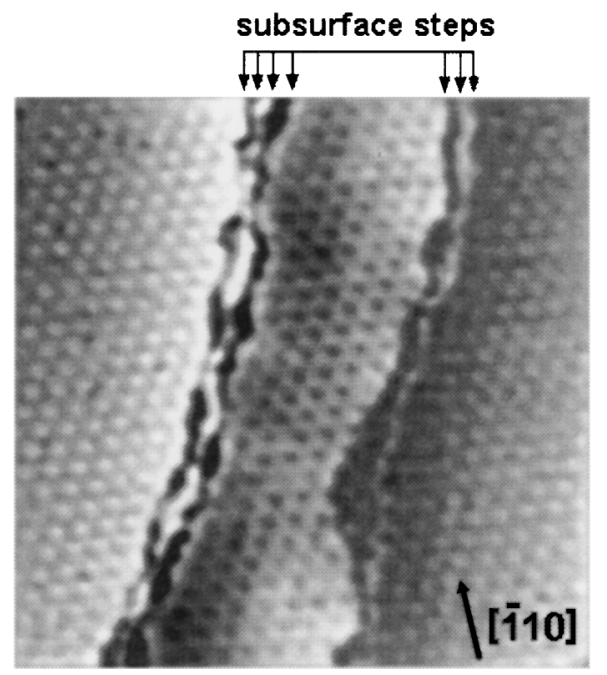

FIG. 2. A typical STM image of a Pb island, obtained at $77 \mathrm{~K}$. The size of the image is $850 \times 850 \AA^{2}$. The superlattice has a period of $40 \AA$ and is rotated by $30^{\circ}$ with respect to $\mathrm{Si}$ [110] axis (indicated by an arrow). For certain metal thickness, the absolute STM contrast is reversed. the orientation and the period of the superlattice remain unchanged. As the absolute contrast of the superlattice exhibits reversals for certain metal thickness (see Fig. 2), this periodic structure cannot be attributed to the surface topology of the $\mathrm{Pb}$ islands.

In our experiments, we examined a large number of $\mathrm{Pb}$ islands, where heights varied from 15 to $100 \AA$ [15]. Nanoscale superlattices were observed in the STM images throughout this thickness range. We found that different $\mathrm{Pb}$ islands often exhibit different types of periodic structures. The three typical examples of such superlattices are shown in Fig. 3. The upper set $(a, b, c)$ and the lower set $\left(a^{\prime}, b^{\prime}, c^{\prime}\right)$ of images in Fig. 3 demonstrate the bias-dependent reversals of the STM contrast. As we found, the STM observation of such superlattices usually requires close alignment of the tip Fermi level, $e U$ (where $U$ is the tip voltage), and the $\mathrm{QW}$ resonance in the $\mathrm{Pb}$ island, $E_{n}$. Moreover, changes in the sign of $e U-E_{n}$ always reverse the contrast of the superlattice in the STM image. A qualitatively similar picture of the resonance-related contrast reversals was reported previously in a STM study of Pb islands on $7 \times 7$ reconstructed silicon, where the data were identified as the remote electronic projection of the buried interface [10]. Therefore, it seems natural to consider the newly observed superlattices as the electronic images of interfacial structures, corresponding to the alternative epitaxial phase of $\mathrm{Pb}$ on $\mathrm{Si}(111)$. As such superlattices have never been observed on a bare $\mathrm{Si}(111)$ surface, their development may be due to only the coalescence of the $\mathrm{Pb}$ and the $\mathrm{Si}$ lattices at their interface. In principle, the development of interfacial structures must be ruled by the minimization of the strain fields. Strain minimization can be achieved by adopting a superlattice, for which the elementary translation vector, $\mathbf{G}$, coincides (or almost coincides) with the nonprimitive 2D translations, $\Sigma n_{i} \mathbf{a}_{i}$ and $\Sigma m_{j} \mathbf{b}_{j}$, of the two interacting
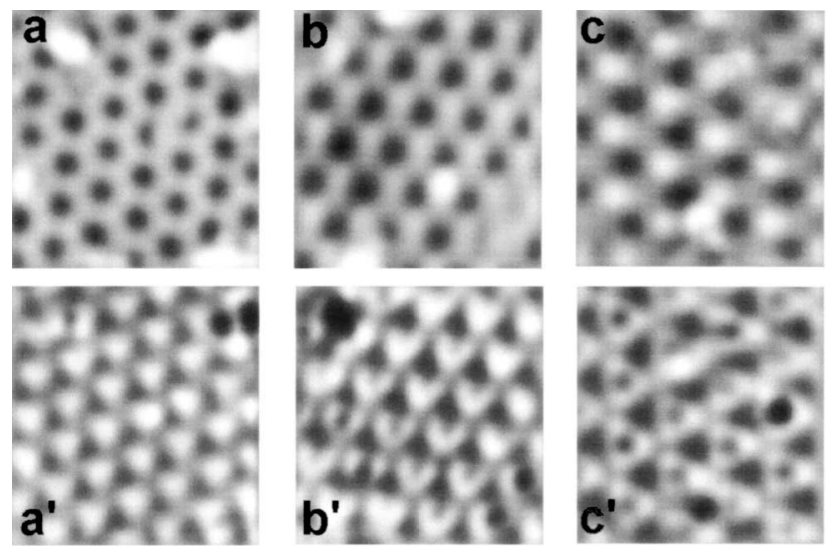

FIG. 3. The $180 \times 180 \AA^{2}$ STM images of three different $\mathrm{Pb}$ islands of $20 \AA$ height. The upper $(\mathrm{a}, \mathrm{b}, \mathrm{c})$ and the lower $\left(a^{\prime}, b^{\prime}, c^{\prime}\right)$ set of images were obtained at tip voltages of -0.45 and $-0.75 \mathrm{~V}$, respectively. $\left(\mathrm{a}, \mathrm{a}^{\prime}\right)$ The period of the superlattice is $33 \AA$. (b, $\left.b^{\prime}\right)$ The period of the superlattice is $43 \AA$. (c, $\left.c^{\prime}\right)$ The period of the superlattice is $40 \AA$. 
lattices (see Fig. 4b). This rule, applied to the $\mathrm{Pb} / \mathrm{Si}$ interface, allows the metallic islands to grow in a few selected orientations [12] (with respect to the substrate), leading to a few types of buried interfacial superlattices.

To obtain more detailed information about these interfacial structures, as well as to find the origin of the biasdependent contrast reversals in the STM images, we performed local density of states (LDOS) measurements. In Fig. 4a, we show the tunneling LDOS spectra, which were measured on the $\mathrm{Pb}$ islands of $55 \AA$ height (upper spectra) and $20 \AA$ height (lower spectra). The energy separation of the QW resonances for these islands equals $\Delta_{1}=0.55 \mathrm{eV}$ and $\Delta_{2}=1.3 \mathrm{eV}$, respectively [16]. For both islands, the spectra were measured at the centers (red spectra) and on the periphery (green spectra) of the supercells. We found that the spectra of the QW resonances exhibit a shift towards lower energies on the
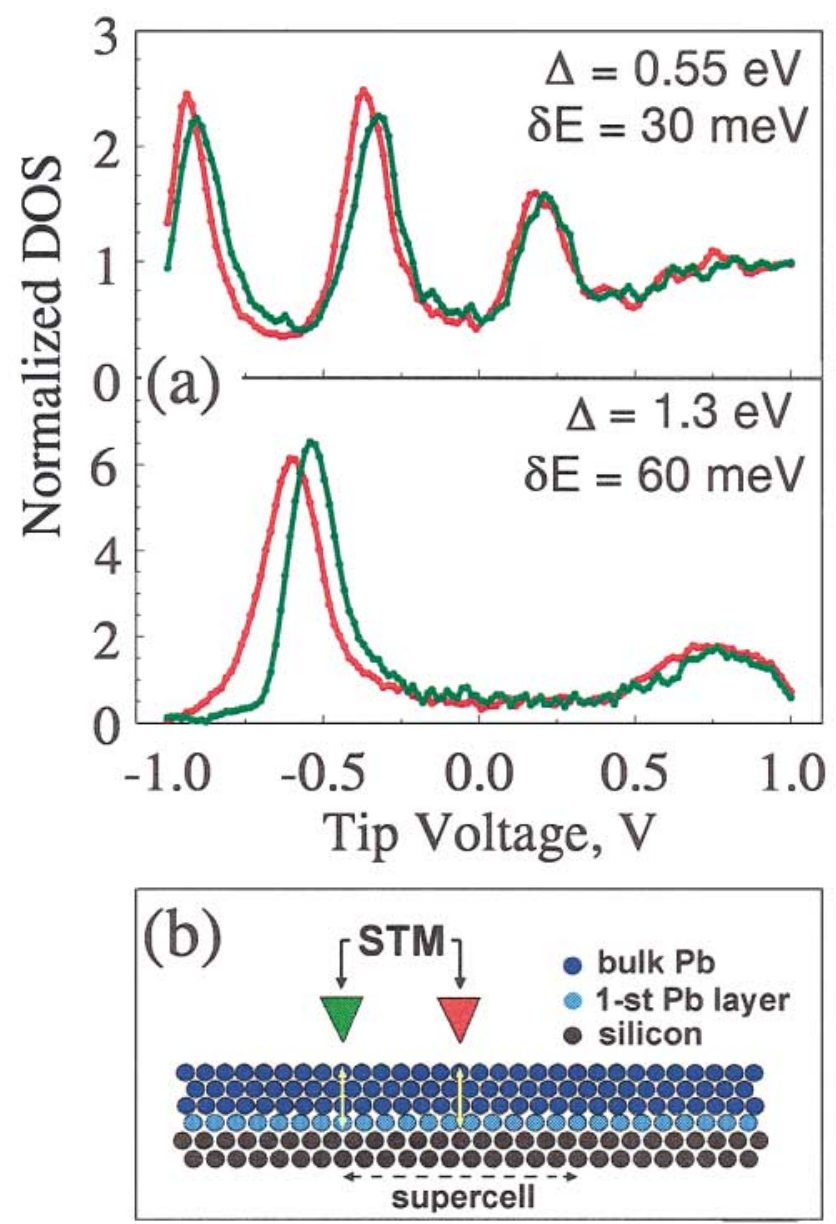

FIG. 4 (color). (a) Tunnel spectra of $55 \AA$ height (upper) and $20 \AA$ height (lower) $\mathrm{Pb}$ islands. The red and the green spectra were measured at the centers and on the periphery of the supercells, respectively. (b) Simplified one-dimensional model, which shows how the relative lateral slip of the $\mathrm{Pb}$ and the $\mathrm{Si}$ lattices affects the electronic reflection phase at the buried interface. The red- and green-marked tip positions correspond to the red and green spectra in (a). periphery of the supercells, which equals $\delta E_{1}=30 \mathrm{mV}$ for the $\mathrm{Pb}$ island of $55 \AA$ height and $\delta E_{2}=60 \mathrm{mV}$ for the $\mathrm{Pb}$ island of $20 \AA$ height.

Thus, unlike in the previously reported study of the buried $7 \times 7$ phase [10], where the subsurface dislocation network suppresses the QW resonances, the annealed $\mathrm{Pb} / \mathrm{Si}(111)$ interface in our experiment causes an adiabatic modulation of their energy positions.

As we show below, both the superlattices in the STM images of the $\mathrm{Pb}$ islands and the lateral modulation of the QW spectra reflect the complex nature of geometric phase in epitaxial nanolayers. First, we consider the requirement of constructive interference in epitaxial $\mathrm{Pb} \mathrm{QW}$

$$
2 K_{z} L+\Phi+\Theta=2 \pi n,
$$

where $K_{z}$ is a normal, [111], component of the wave vector, $L$ is the QW thickness, whereas $\Phi$ and $\Theta$ are the electron reflection phases, associated with the metal-vacuum and the metal-semiconductor interfaces, respectively. This requirement yields a discrete set of allowed values of $K_{z}$, such that

$$
K_{z}=\pi n / L-(\Phi+\Theta) / 2 L .
$$

Since the top surface of the $\mathrm{Pb}$ islands is a flat (111) crystal face, we can assume $\Phi=$ const. By contrast, the structure of the buried $\mathrm{Pb} / \mathrm{Si}$ interface is inhomogeneous at the nanometer scale, so that the reflection phase becomes a function of lateral position, $\Theta=\Theta(\mathbf{r})$, where $\mathbf{r}=(x, y)$. Such nanoscale inhomogeneities are likely related to the interfacial deformation fields, as discussed earlier. As the reflection phase $\Theta$ changes, so do the allowed values of $K_{z}$, which in turn shift the QW energy spectra

$$
\delta E(\mathbf{r}) \approx \hbar \nu_{f} \delta K_{z}=-\hbar \nu_{f} \frac{\delta \Theta}{2 L},
$$

where $\nu_{f}$ is the Fermi velocity. Taking into account the well-known expression for the energy separation between the QW resonances, $\Delta=\pi \hbar \nu_{f} / L$, Eq. (3) can be rewritten as

$$
\delta E(\mathbf{r}) \approx-\frac{\Delta}{2 \pi} \delta \Theta(\mathbf{r}) .
$$

With Eq. (4), we can now explain both the origin of the energy shift, $\delta E$, observed in tunneling spectra of the $\mathrm{Pb} \mathrm{QW}$, and the increase of this spectral shift for larger $\Delta$ (thinner $\mathrm{QW})$. As our data suggest, the peak-to-peak lateral changes of the reflection phase $\delta \Theta=0.31( \pm 10 \%)$. The simplified one-dimensional model, presented in Fig. $4 \mathrm{~b}$, demonstrates how the relative lateral slip of the $\mathrm{Pb}$ and the Si lattices changes the detailed atomic arrangement at the interface [17]. The comparison of our data with Eq. (4) suggests the increase of $\Theta$ on the periphery of the supercell, which may be related to the antiphase alignment of the $\mathrm{Pb}$ and the Si lattices. Furthermore, one should not exclude the possibility of a transversal modulation of the position of $\mathrm{Pb}$ atoms at the interface, which is not shown 
in Fig. 4b. Such "height" modulations $(\sim 0.1 \AA)$ will also contribute to the reflection phase.

As has been indicated in a series of theoretical works [2-4], in quantized systems with adiabatic boundary inhomogeneities, the lateral electron motion can often be described by means of effective boundary-induced potentials. In thin $\mathrm{Pb}(111)$ films, the adiabatic shift of QW resonances, $\delta E(\mathbf{r})$, which we observed in our experiments, may be considered such an effective 2D potential. Although this potential is rather "shallow" $(\delta E \ll \Delta)$, for heavy $2 \mathrm{D}$ electrons in $\mathrm{Pb}(111) \mathrm{QW}$ it can yield a noticeable effect. This effect may become especially strong in ultrathin $\mathrm{Pb}$ films, as seen in Fig. $4 \mathrm{a}$, where $\delta E$ becomes comparable to the $2 \mathrm{D}$ bandwidth. In such a case, one should expect a transformation of a thin $\mathrm{Pb} \mathrm{QW}$ into an array of 2D quantum dots.

In conclusion, scanning tunneling microscopy and spectroscopy of epitaxial $\mathrm{Pb}$ islands on $\mathrm{Si}(111)$ reveal adiabatic lateral modulation of the QW energy spectra, providing remote electronic images of the subsurface reflection phase. As we show in this Letter, localization caused by FS nesting (transverse backscattering) can be further enhanced by lateral gradients of the boundary reflection phase. Since many materials exhibit FS nesting, this phenomenon should be common to a variety of heteroepitaxial systems.

We thank I. Shalish, R. E. Martinez II, C. H. Perry, and I. Appelbaum for fruitful discussions. The research was supported by the Rowland Institute for Science and NSF.
[1] J. J. Paggel, T. Miller, and T.-C. Chiang, Science 283, 1709 (1999).

[2] Z. Tesanovic, M. V. Jaric, and S. Maekawa, Phys. Rev. Lett. 57, 2760-2763 (1986).

[3] A. E. Meyerovich and S. Stepaniants, Phys. Rev. Lett. 73, 316 (1994); Phys. Rev. B 60, 9129 (1999).

[4] A. Kawabata, J. Phys. Soc. Jpn. 62, 3988 (1993).

[5] M.E. Rubin et al., Phys. Rev. Lett. 77, 5268 (1996).

[6] M. Pratzer et al., Phys. Rev. Lett. 87, 127201 (2001).

[7] L. Vitali, M. G. Ramsey, and F. P. Netzer, Phys. Rev. Lett. 83, 316 (1999).

[8] I. B. Altfeder, K. A. Matveev, and D. M. Chen, Phys. Rev. Lett. 78, 2815 (1997).

[9] S. Heinze et al., Phys. Rev. Lett. 83, 4808 (1999).

[10] I. B. Altfeder, D. M. Chen, and K. A. Matveev, Phys. Rev. Lett. 80, 4895 (1998).

[11] J. R. Anderson and A. V. Gold, Phys. Rev. 139, A1459 (1965).

[12] H.H. Weitering, D. R. Heslinga, and T. Hibma, Phys. Rev. B 45, 5991 (1992).

[13] I.-S. Hwang et al., Phys. Rev. B 51, 10193 (1995).

[14] L. Seehofer et al., Phys. Rev. B 51, 13503 (1995).

[15] The island heights are measured from the top of the $\mathrm{Pb}$ wetting layers. These two $\mathrm{Pb}$ layers also contribute to the QW thickness.

[16] The parameter $\Delta$ indicates the separation of the LDOS maxima, which correspond to the lowest unoccupied and the highest occupied QW states.

[17] Because of a rotation of the $\mathrm{Pb}(111)$ plane with respect to the $\mathrm{Si}(111)$ plane the actual atomic arrangement becomes more complicated. 